\title{
A Low-Power Microcontroller with Accuracy-Controlled Event-Driven Signal Processing Unit for Rare-Event Activity-Sensing IoT Devices
}

\author{
Daejin Park, ${ }^{1}$ Jonghee M. Youn, ${ }^{2}$ and Jeonghun Cho' \\ ${ }^{1}$ School of Electronics Engineering, Kyungpook National University, 80 Daehakro, Bukgu, Daegu 702-701, Republic of Korea \\ ${ }^{2}$ Department of Computer Engineering, Yeungnam University, 280 Daehak-Ro, Gyeongsan, Gyeongbuk 712-749, Republic of Korea \\ Correspondence should be addressed to Jonghee M. Youn; youn@yu.ac.kr and Jeonghun Cho; jcho@ee.knu.ac.kr
}

Received 8 January 2015; Accepted 23 March 2015

Academic Editor: Young-Sik Jeong

Copyright ( $\odot 2015$ Daejin Park et al. This is an open access article distributed under the Creative Commons Attribution License, which permits unrestricted use, distribution, and reproduction in any medium, provided the original work is properly cited.

A specially designed microcontroller with event-driven sensor data processing unit (EPU) is proposed to provide energy-efficient sensor data acquisition for Internet of Things (IoT) devices in rare-event human activity sensing applications. Rare-event sensing applications using a remotely installed IoT sensor device have a property of very long event-to-event distance, so that the inaccurate sensor data processing in a certain range of accuracy error is enough to extract appropriate events from the collected sensing data. The proposed signal-to-event converter (S2E) as a preprocessor of the conventional sensor interface extracts a set of atomic events with the specific features of interest and performs an early evaluation for the featured points of the incoming sensor signal. The conventional sensor data processing such as DSPs or software-driven algorithm to classify the meaningful event from the collected sensor data could be accomplished by the proposed event processing unit (EPU). The proposed microcontroller architecture enables an energy efficient signal processing for rare-event sensing applications. The implemented system-on-chip (SoC) including the proposed building blocks is fabricated with additional 7500 NAND gates and 1-KB SRAM tracer in 0.18 um CMOS process, consuming only $20 \%$ compared to the conventional sensor data processing method for human hand-gesture detection.

\section{Introduction}

Nowadays, sensor devices with wireless connectivity such as $\mathrm{Wi}-\mathrm{Fi}$, Bluetooth, and ZigBee are becoming important in IoT applications. Human activity monitoring based on sensed signal analysis $[1,2]$ is becoming a popular application using biomedical computing technology. Body-attached sensors measure the electrical signal converted by a biomedical interface [3]. Observation-based remote sensors are also used to monitor the movement of the human body [4]

As described in Figure 1, the remotely installed smart sensor system monitors the environment periodically and transfers the collected sensor data via wireless interface to the host system such as a Web server. These wireless sensor devices are powered by the on-board battery with limited capacity.

Battery recharging and replacement are very inconvenient procedures and a major obstacle in extending various IoT-based applications. Long operating lifetime of the sensor systems is therefore an important requirement in designing the system architecture and the sensor data processing algorithm.

The energy consumption in the sensor systems is caused by sampling the sensor signal, processing the sampled data, and transferring the collected data to the host machine via wireless interface. Traditional sensor devices sample the signal periodically and analyze the sensed data.

This approach has advantages requiring the simple processing unit in terms of hardware resource and the generalized algorithm in terms of software development. Syntactic activation of the entire sensor system results in more power consumption, especially in wireless connectivity.

The conventional sensing platform for human activity monitoring uses general purpose microcontrollers (MCUs), including an analog sensor interface, discrete-time analog-todigital converter (ADC) as a data sampler, and a sensor data processing unit to analyze collected data. 


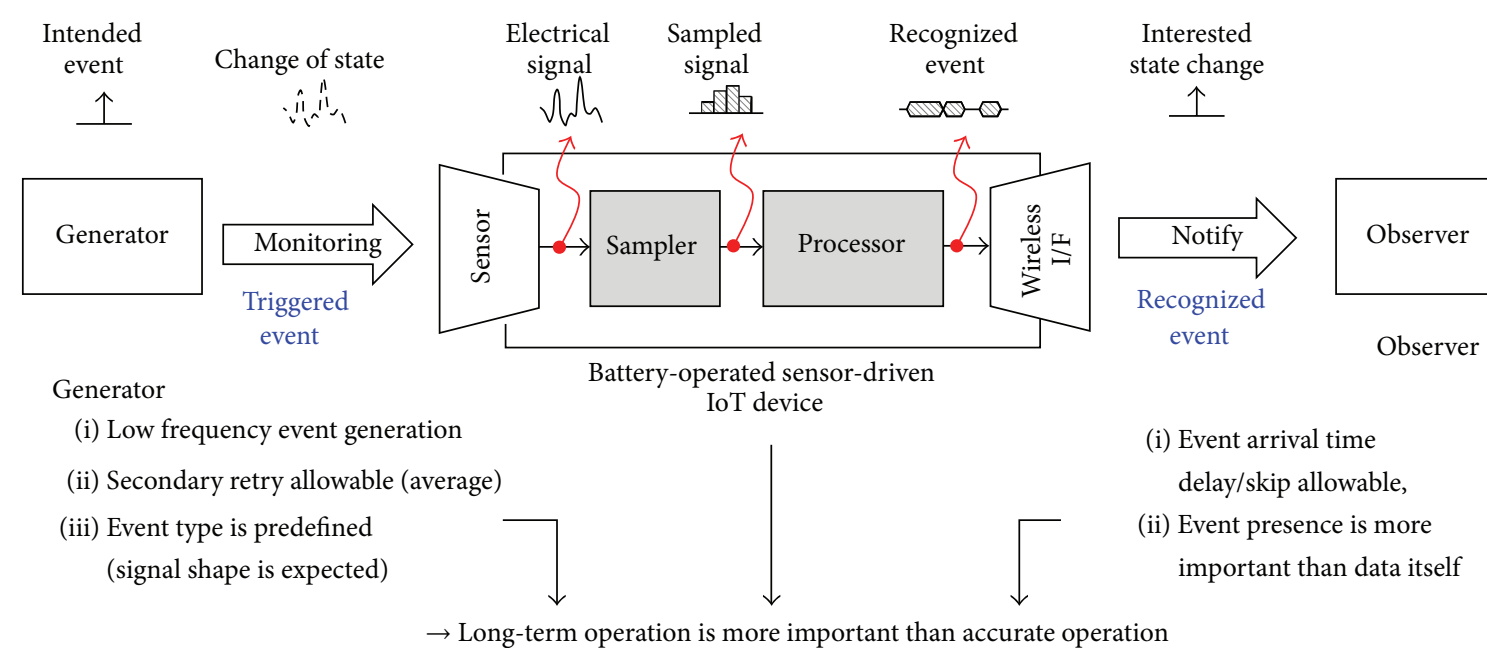

FIGURE 1: Sensor-based IoT device concept with sensor interface, processing unit, and wireless connectivity.

However, this approach, by which the sensed data is analyzed on the microlevel of data-to-data, has operating power overhead because it is not optimized to consider the long event-to-event distance of human activity signals, which is easily observed in rare-event sensing applications $[4,5]$.

In this paper, for efficient sensor data processing in the energy consumption, a semantic sampling method is introduced to capture the signal with the features of interest and is implemented as a preprocessor unit named to signal-to-event converter (S2E), which generates the atomic events instead of the sampled data itself. The extracted atomic events are a relatively small number of samples compared to the syntactic sample data by conventional analog-to-digital converter (ADC) as a signal-to-data converter (S2D).

The proposed S2E replaces the conventional S2D to extract atomic events from the incoming sensor signal. The event identification from human activity monitoring is performed by the event-driven sensor data processing unit (EPU) for the small set of extracted atomic events.

This paper is organized as follows. In Sections 2 and 3 , our research motivation and related work are discussed. Section 4 describes the details of the proposed architecture. The implementation and experimental results are presented in Section 5. Finally, we conclude the paper by summarizing our contributions in Section 6.

\section{Motivation}

The key motivation of the proposed method begins with the transition to macrolevel processing of the sensor signal by S2E instead of the conventional microlevel analysis for the sensed dataset. Figure 2 shows the method we used to represent the sensor signal acquisition result in the event data space. The human activity sensor signals are represented by the attributes of interest and the elapsed time relationship between the atomic events in Figure 2(a). The monitored human activity is encoded as a set of atomic events by the event quantization in Figure 2(b).
To address this limitation of the conventional digital system architecture by using the discrete time-based sensor data processing method, we propose an event-driven system architecture that modifies traditional digital system design. We present a theoretical framework to implement an eventdriven sensor processor for general rare-event sensing applications by analyzing the system operations.

2.1. Event-Space Signal Representation. Our main research begins with an event-space representation of the signal, instead of the digital data space domain. The extracted features of the sensed signal are encoded into the elapsed time between events and informative value such as voltage level and edge phase crossing the trigger point of the signal. The fundamental event defined, which is defined as an atomic event with the most important information, provides a signal representation on an abstract level and reduces the computational complexity in performing basic data processing for extracted informative features of interest. The collected atomic events include partial information in the original signal that specifies whether the desired featured points of the signal are present.

2.2. Accuracy-Controlled Event Quantization. The eventquantization concept extends the time-quantization method for signal representation that uses elapsed time to enhance the conventional data-sampling and processing method. Time quantization monitors only the specific conditions of the signal transition and captures the time-stamps. The eventquantization method also determines whether the specified characteristics of the signal exist.

2.3. Event-Driven Sensor Data Processing. The event-based approach, with a certain amount of accuracy error, is described by the proposed event-driven sensor data processing flow. The input signal is monitored with specified interest-ofsignal characteristics to generate the specific atomic events of the signal. The set of atomic events during the specified region 
Human activity signal acquisition and event quantization

$$
\underset{\text { Target }}{=} \Rightarrow \sqrt[f]{ } \Rightarrow
$$

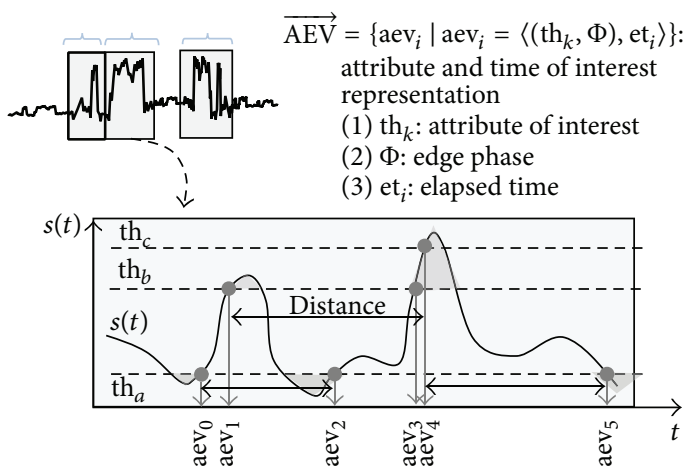

(a) Attribute and its corresponding elapsed time representation
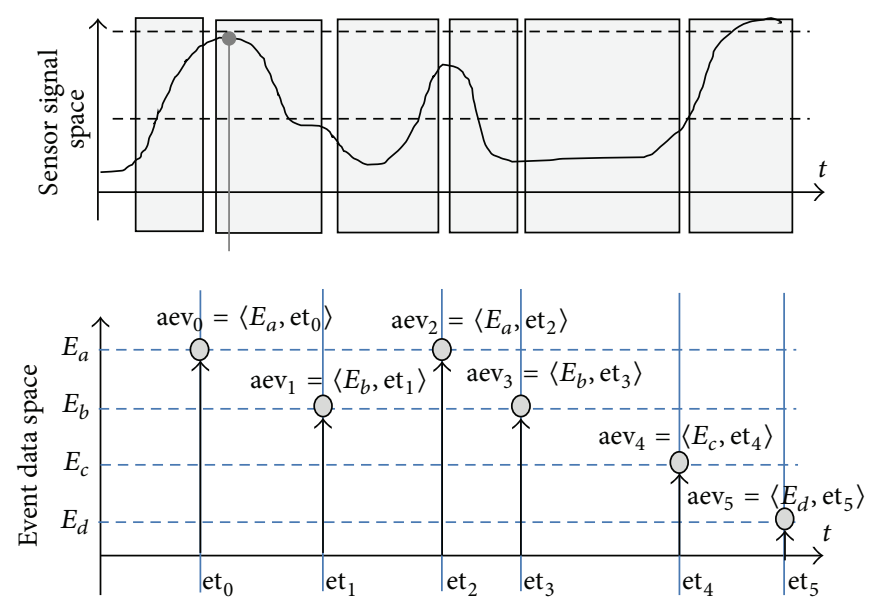

(b) An example of event-space representation for incoming sensor signal

FIGURE 2: Human activity sampled data representation in event data space.

of the signal are traced into the tracer memory as an event vector, which contains the sequence of the atomic events and the time-distance relationship between the atomic events. The traced event vector identifies the approximate result as a final event by comparing it to the expected rules of the atomic events.

These approximation approaches enable us to reduce the computational complexity in order to manipulate a large amount of collected sensing data. As a result, power consumption will be reduced. For applications related to human interaction, an approximation approach enables developers to design the computational block using smaller hardware resources, while providing sufficient performance in limited resolution of the accuracy.

In accuracy-controlling approaches defined from the specifications, our study focused on the data-representation resolution, the timing-resolution of the sampling frequency, and the response time as a delay time [6]. This enables the configuration of the operation accuracy in the processor architecture level according to the abstraction level of the proposed event-quantization approach.

\section{Related Work}

To overcome the weakness of inefficient power consumption by the frequent CPU wake-up for the discrete time sampling, continuous-time signal processing techniques $[7,8]$ have been proposed in previous literature. If a certain condition of the signal status, such as the voltage level at a specific time, is matched with the user-defined condition $[9,10]$, the time value at the triggered condition is sampled and quantized [5] by the selective method, which also helps to reduce operational power [11].

The continuous-time sampling method was introduced to improve the syntactic sampling and processing approach in terms of power consumption, but it requires additional hardware resources and more computational time for the timedistance calculation, which gives rise to additional power consumption. The required power and hardware resource overhead, which are needed to compensate for reduced wakeup power consumption, must be considered in order to achieve benefits in total energy efficiency due to hardwareenergy trade-off.

The trade-off in terms of energy and accuracy has been studied widely $[12,13]$. To obtain long lifetime operations under limited battery power [14], the latest research introduces inaccurate computation techniques $[15,16]$ with approximation-based hardware designs.

The proposed sensor processor for the rare-event sensing applications adopts the event-driven approach of the continuous-time sampling method. Inaccurate time-data manipulation reduces computational complexity and sampling resolution by determining the presence of featured events in the specific range. The event-detection accuracy can be adjusted by making the trade-off between the processing energy consumption and the operating specification.

\section{Proposed Architecture}

4.1. Application-Dependent Constraints. Figure 4 shows the difference between the discrete time samples and the featured events of interest, with the common shape of the rare-event sensor signal. Event sources, such as hand gestures, proximity, and object activity, generate signal pulses for which the distance between featured points of the signal is very long. The number of data samples $(n)$ is greater than the number of events $(m)$. In this work, we assumed an applicationspecific constraint of rare-event characteristics, which result in a small number of events compared to the number of data samples. 


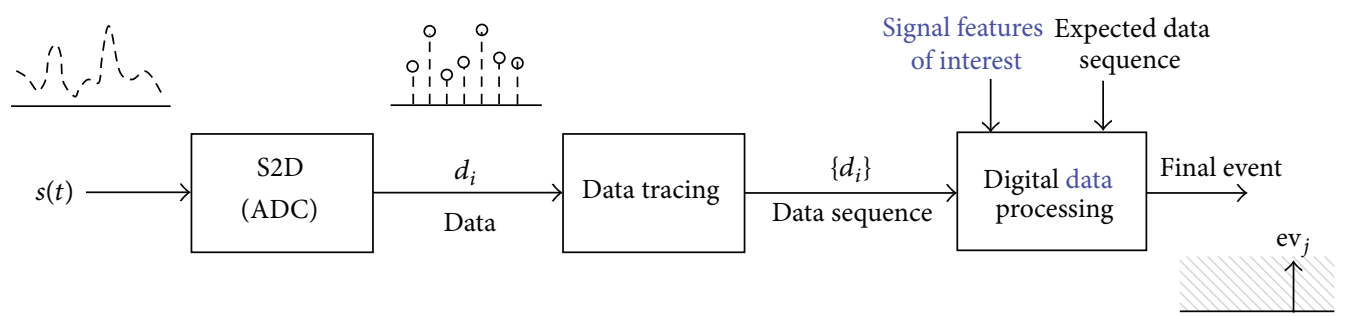

(a) Data sampling and lazy evaluation for syntactic data processing

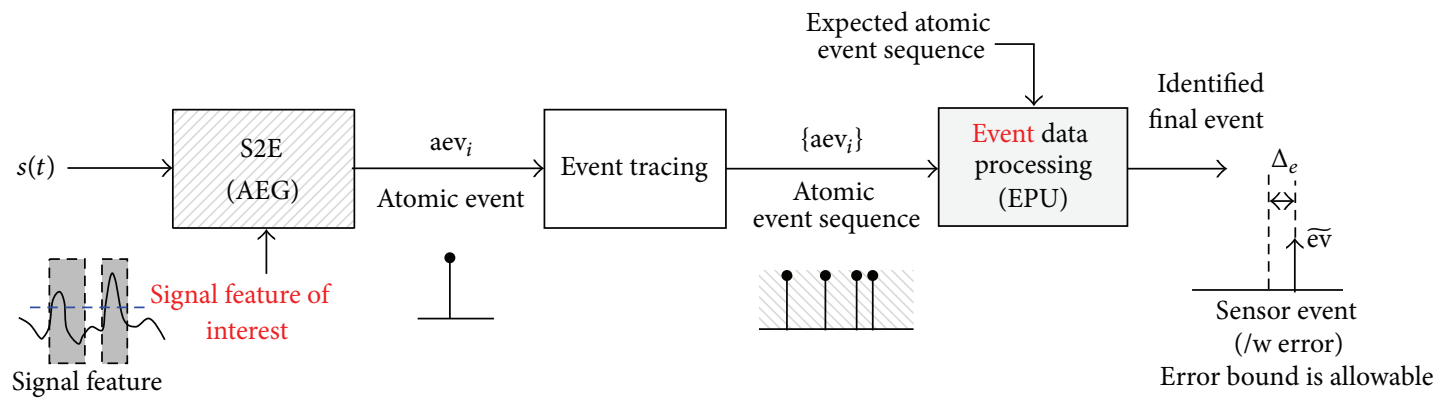

(b) Event sampling by early evaluation and event processing

FIGURE 3: Event sampling based on signal-to-event (S2E) and event-driven data processing.

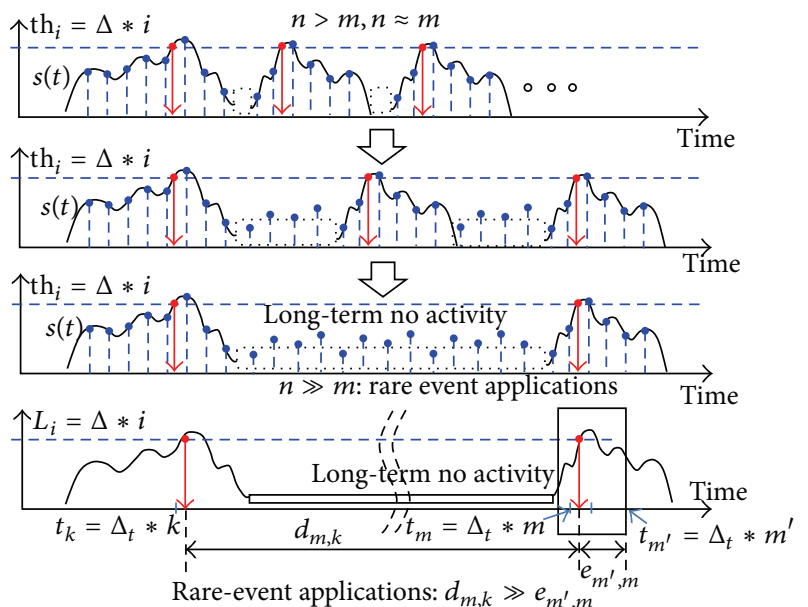

FIGURE 4: Wake-up frequency for data sampling and event-driven sampling.

The event-quantization accuracy depending on the resolution of the elapsed time-stamp is described as $e_{m^{\prime}, m}$ in Figure 4 . The rare-event sensing applications, in which the event-to-event duration is relatively larger than the accuracy error, have the following application-specific constraints:

$$
d_{m, k} \gg e_{m^{\prime}, m}
$$

With these application-specific constraints in (1), the event identification accuracy error caused by the inaccurate time-stamp measurement clock is relatively insensitive. The recognized event observer, such as the human eye, allows for a certain amount of inaccuracy in identifying the meaning of the events, which are constructed by the proposed inaccurate event-driven sensor processor.

The proposed sensor processor is designed with these application-specific constraints by reducing the accuracy of the time-stamp measurement clock, decreasing the bit width of the timer block to capture the time-stamps, and decreasing the operational complexity of the time-to-time distance measurement blocks, which are specially implemented as a dedicated accelerator for event recognition in the implemented hardware.

4.2. Atomic Event Quantization. The conventional MCU performs data sampling in the ADC unit, data tracing in buffer memory, and digital data processing to identify the original event generated by event sources, such as a swipe gesture. The syntactic sampling is performed without the consideration of the incoming signal property. Then, the lazy evaluation using the features of interest is performed to generate the final event $\mathrm{ev}_{i}$ using a large number of sampled data $\left\{d_{i}\right\}$. This syntactic data sampling and lazy evaluation in conventional MCU is illustrated in Figure 3(a).

The proposed EPU can perform the event relationship analysis with a reduced computation overhead for the smaller set of atomic events. The signal abstraction by extracting atomic events as signal shape in S2E leads to accuracy error in identifying the final event. The overall procedure of the event-driven processing in the modified MCU is described in Figure 3(b).

The event-driven signal sampling in the proposed architecture captures the signal shapes of interest using the feature scanning window, which determines the presence of the expected features of the signal. The feature scanning window in Figure 5(a) is configured to capture the specific signal 
Configuration of feature scanning window

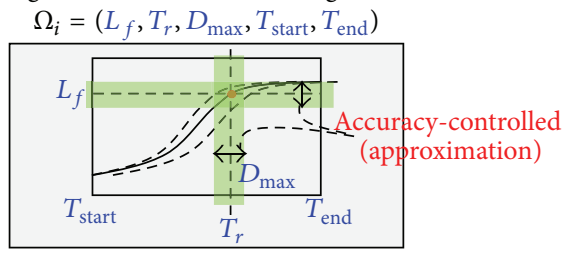

(a) Configuration of signal scanning window for atomic event extraction

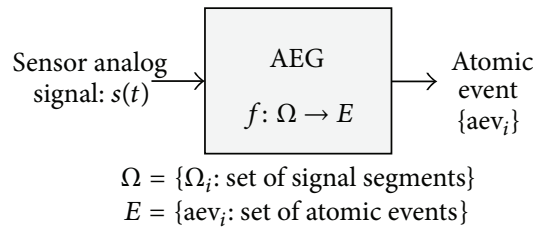

(b) Atomic event generator definition

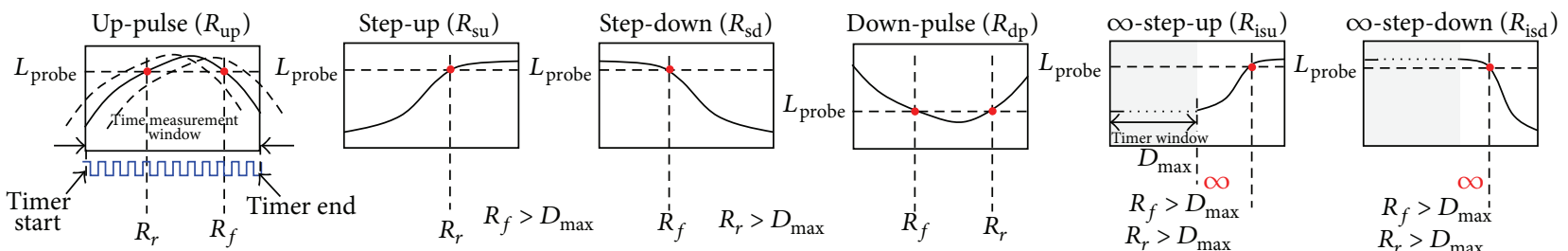

(c) Examples of set of signal segment $\Omega$
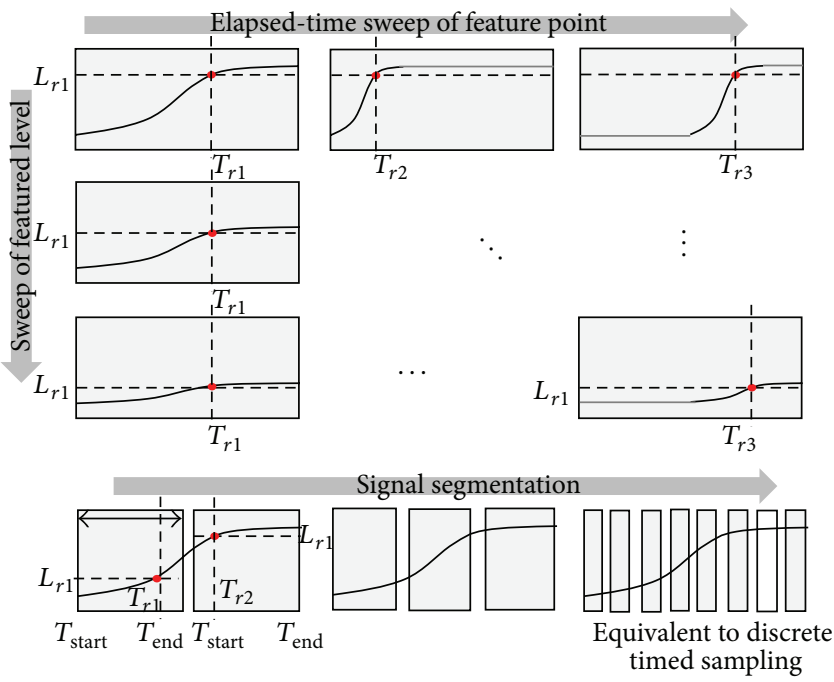

(d) Representing various atomic events according to featured points

FIGURE 5: Atomic event generator (AEG) based on feature scanning window and signal segmentation.

shape. This configuration is represented with the set of signal segments $\Omega$ in Figure 5(b).

The S2E includes the atomic event generator (AEG) unit to generate a set of atomic events by using the user-defined set of signal segments $\Omega$. Examples of the user-defined signal segments $\Omega$ are introduced in Figure 5(c).

Figure 7 describes our approach to capture the signal shape as an atomic event crossing a certain range of arrival time. To more formally define our approach, we begin our explanation by first presenting the following definitions.

Definition 1. Given continuous signal $s(t)$, let AEV $=\left\{\mathrm{aev}_{i} \mid\right.$ $\mathrm{aev}_{i}=\left(\mathrm{aev}_{i-1}\right.$, value, phase, et $\left.)\right\}$ be a sequence of an atomic

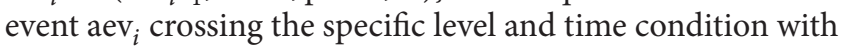
a relationship of previous atomic event $\mathrm{aev}_{i-1}$, where $\mathrm{aev}_{i}$. value is a result of the approximation-based data quantization function ADQ and $\mathrm{aev}_{i} \cdot$ et is a result of the approximationbased time quantization function ATQ, described as follows:

$$
\begin{gathered}
\widetilde{d_{k}}=\operatorname{ADQ}\left(s\left(t_{k}\right), L_{m}, \Delta_{d}, u, v\right), \\
\forall \Delta_{d} * u<\left|L_{m}-d_{k}\right|<\Delta_{d} * v \\
\widetilde{t_{k}}=\operatorname{ATQ}\left(\operatorname{aev}_{i} \cdot \text { et } \widetilde{T_{\mathrm{clk}}}\right), \quad \text { where } \widetilde{T_{\mathrm{clk}}}=T_{\mathrm{clk}}+\Delta_{t} .
\end{gathered}
$$

The meet condition $k$, when the expected crossing is present, is described in the following equation:

$$
\widetilde{t_{k}}=\mathrm{et}+\widetilde{T_{\mathrm{clk}}} * k, \quad \forall \mathrm{DQ}\left(s\left(\widetilde{t_{k}}\right)\right)=\widetilde{d_{k}} \text {. }
$$

As shown in Figures 5(a) and 5(b), the AEG builds an element with the attributes, which are encoded with the digitized 

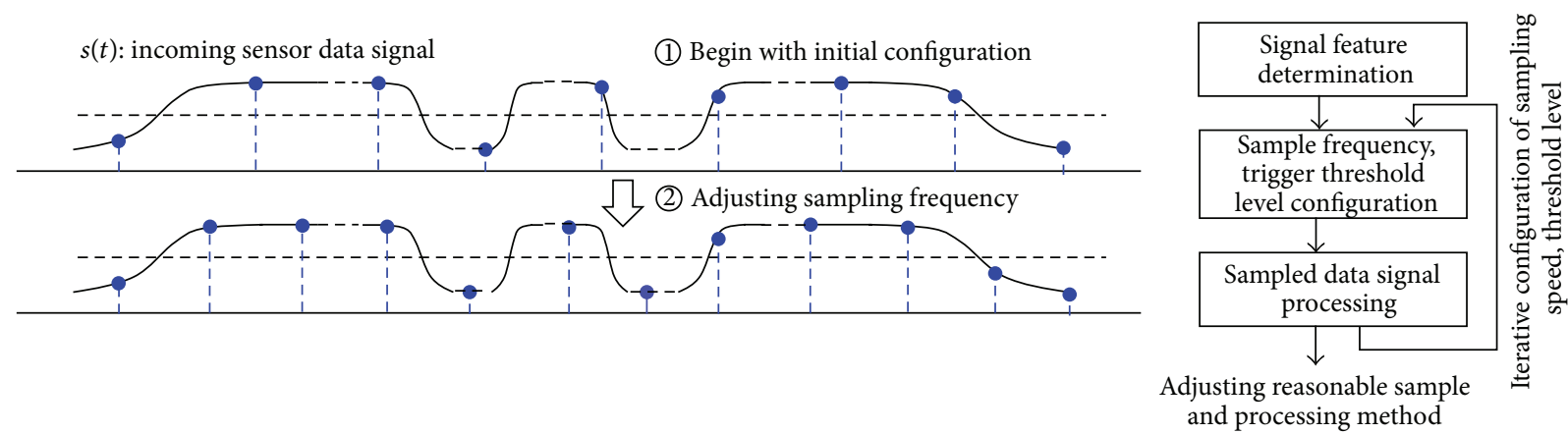

(a) Syntactic procedure to determine conventional data sampling frequency

(1) Begin from fundamental atomic event set

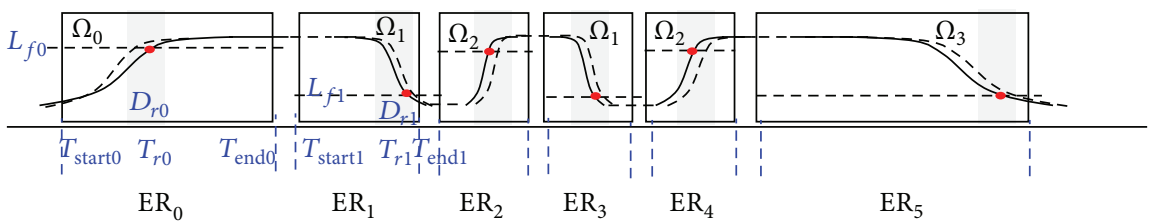

П (2) Extend the event sampling window by grouping the adjacent event
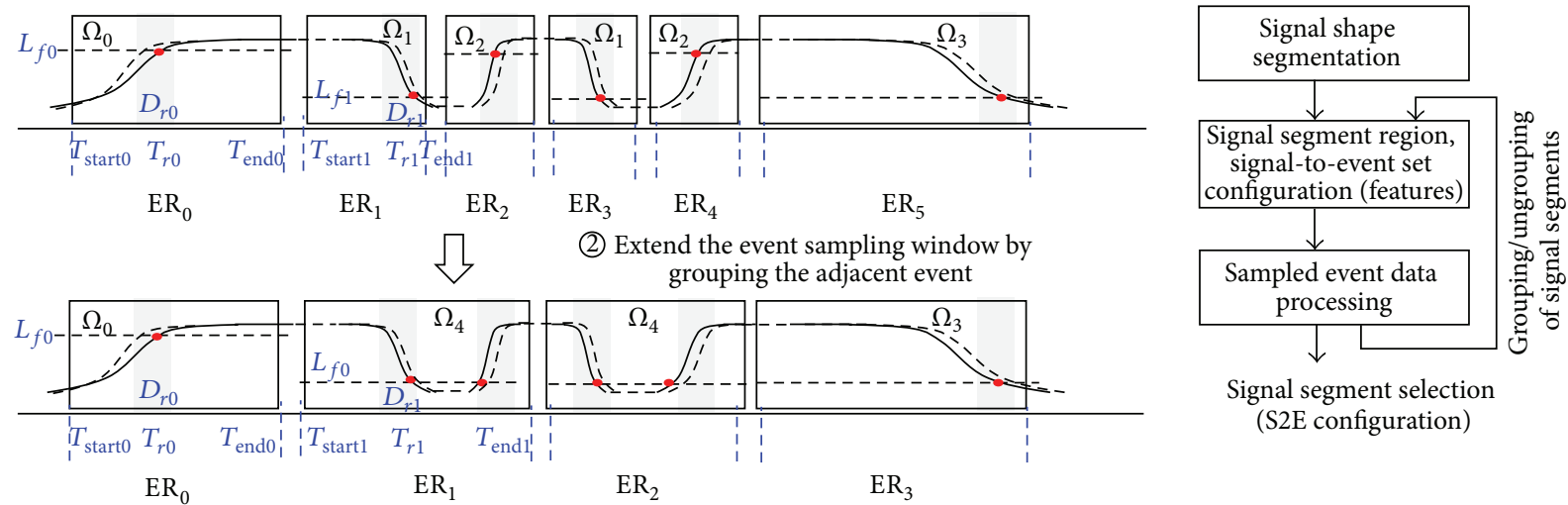

(S2E configuration)

(b) Iterative procedure to determine appropriate event segmentation set

FIGURE 6: Iterative procedure to determine sampling method and signal segments for the sensor signal.

signal level, elapsed time, and edge phase in the following equation:

$$
\operatorname{AEG}(s(t), L)=\left\{\operatorname{aev}_{i} \mid \operatorname{aev}_{i}=\left\langle\operatorname{aev}_{i-1}, \widetilde{d_{k}}, \phi_{\text {edge }}, \widetilde{t_{k}}\right\rangle\right\} .
$$

From (4), the extracted information, as an atomic event, is encoded with the approximation value of the signal level, the reduced time quantization value of the elapsed time, and the relationship of the previous atomic event $\mathrm{aev}_{k-1}$.

4.3. Atomic Event Extraction. The event-quantized signal representation is dependent on the event slice resolution of the configured set of signal segments, which is described in Figure 5(d). The number of feature points and the window size determines the accuracy of the signal representation by the extracted atomic events. Figure 5(d) shows the capability to represent various signal shapes with the configuration of $L_{r}, D_{\max }, T_{r}, T_{\text {start }}, T_{\text {end }}$ in the feature scan window.

Definition 2. Given the configured feature scanning window to extract the atomic events from $s(t)$, let $T_{\text {start }}$ be a start time monitoring the signal, let $T_{\text {end }}$ be the end of monitoring the signal, let $L_{r}$ be a rising signal level at which the time-stamp is $T_{r}$, let $L_{f}$ be a falling signal level at which the time-stamp is $T_{f}$, let the pair of $L_{x}$ and $T_{y}$ be featured point, and let $D_{\max }$ be a maximum time value in which the featured points are present. The set of signal segments described by the configuration $\Omega=\left\{\Omega_{i} \mid \Omega_{i}=\left(T_{\text {start }}, T_{\text {end }}, L_{r}, L_{f}, T_{r}, T_{f}, D_{\max }\right)\right\}$ of the featured scanning window is defined as $\Omega$, and they are used to extract the atomic events of interest for the AEG function, which is defined as follows:

$$
\left\{\operatorname{aev}_{i}\right\}=\operatorname{AEG}(s(t), \Omega) .
$$

$\Omega_{\text {up }}$ defines a signal segment of the feature scanning window with the "up-pulse" type in the first part of Figure 5(c). In our applications, $\left\{\Omega_{\text {type }} \mid\right.$ type = "up", "su", "sd", "dp", "isu", "isd" $\}$ is used.

One signal shape can be divided into several slices by user-defined signal segmentation. If the time window for signal segmentation is the same as the fixed width $t_{s}$ in the discrete time sampling method, the result of the atomic event generation is equivalent to that of the discrete time sampling. The proposed atomic event generation approach enables a trade-off between the signal extraction accuracy and its processing power consumption.

The application-specific constraints in configuring the set of signal segments must be considered for the accuracyenergy trade-off to provide reasonable accuracy of event identification with limited energy consumption. Figure 6 shows the determination procedure of the signal segments to represent the sensed signal with small set of signal segments. A reasonable slice of the signal segmentation can be 


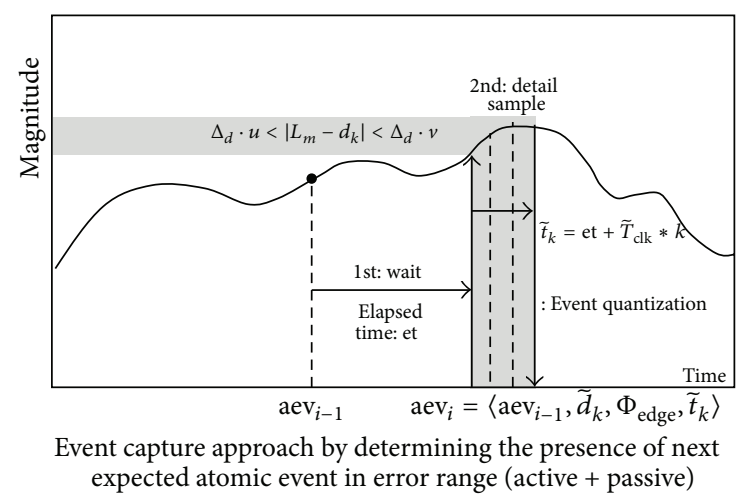

FIGURE 7: Event sample by capturing the specific features of interest and elapsed time.

determined by the iterative configuration to provide enough event identification performance with reasonable energy consumption.

Figure 6(a) shows an example of searching reasonable sampling frequency. The red colored sample can be obtained by adjusting the sampling frequency after the specified activity signal is analyzed. Figure 6(b) describes the procedure of grouping a set of signal segments into another signal segment, which can represent the activity signal with a smaller number of atomic events.

4.4. Event-Driven Sensor Data Processing. The AEG scans the continuous signal $s(t)$ passing through the configured feature scan window to determine the presence of the signal shapes of interest, as shown in Figure 8. The set of atomic events is generated with a pair of attributes and time-stamps as a result of the time quantization shown in Figure 9:

$$
\operatorname{aev}=\left\{\operatorname{aev}_{i} \mid \operatorname{aev}_{0}, \operatorname{aev}_{1}, \ldots, \operatorname{aev}_{i}=\left(“ L_{i} ", t s_{i}\right)\right\} .
$$

The signal representation by a set of atomic events with a certain amount of error is denoted in the following equation:

$$
\widetilde{\mathrm{ae}}=\left\{\widetilde{\mathrm{ae}_{i}} \mid \widetilde{\mathrm{ae}_{0}}, \widetilde{\mathrm{ae}_{1}}, \ldots, \widetilde{\mathrm{ae}_{i}}=\left(“ L_{i}, \overline{t s_{i} \pm \Delta}\right)\right\} .
$$

$\mathrm{aev}_{i}$, which is matched with the configured scan window $\mathrm{AE}_{i}$, is represented as an abstracted atomic event index in Figure 9, which indirectly addresses the detailed attributes in the constant dictionary. The continuous analog signal is converted into a set of event quantized data $\widetilde{\operatorname{aev}}_{i}$, and its index value is traced only into the atomic event tracer buffer. Therefore, the traced event data processing manipulates the index value and its relationship to the representative atomic events to generate the final event EV. The proposed EPU, which is based on event quantization, provides the following advantages compared to conventional sensor data processing.

4.5. Event Bus Architecture. The modified architecture of the proposed MCU includes S2E to extract atomic event aev from the activity signal instead of using ADC, event tracing to archive the atomic events $\left\{\mathrm{aev}_{i}\right\}$, and the EPU to analyze the relationship between the archived atomic events.
The sensor signal in rare-event applications is described with an example in Figure 2(a), which is represented with the threshold level, edge phase type, and elapsed time between the previously recognized signal points. The signal features of interest are used early to extract the atomic events in the S2E unit.

The path from S2E to the event tracer is designed with the event bus, on which the atomic event transactions are loaded. The predefined event types are configured in EPU configuration by the user knowing the signal characteristics for which attributes are represented. The EPU handles the index to the events in the event table, which is stored in the EPU configuration. Figure 9 shows data flow of the eventquantized atomic events. The atomic event $\operatorname{aev}_{i}$ only contains a pointer to address the detailed attributes in the attribute table and elapsed time table to save the limited tracer memory area.

\section{Implementation and Experimental Results}

Figure 10(a) is the data path of the implemented S2E circuit. The proposed S2E-based signal conversion and event signal processing architecture requires additional hardware overhead, including a level comparator, AEG, timer, tracer memory, and EPU, which are distinguished with a red dotted line in Figure 10(b).

The hardware implementation based on the proposed concept requires the additional 7500 NAND gates and $1 \mathrm{~KB}$ SRAM tracer in 0.18 um CMOS process. The implemented designs are integrated in an 8051-based microcontroller. Figure 10(b) shows the modified event-bus architecture of the implemented MCU, in which the atomic event $\left(\mathrm{aev}_{i}\right)$ is loaded from S2E. The attributes of the user-defined atomic event, including signal features and elapsed time ranges, are stored as a constant table in the on-chip flash memory.

For power consumption measurement, the raw dump of the electrical signal generated by hand gesture is gathered into the host computer, as shown in Figure 10(c). The input stimulus of the activity signal is loaded into the circuit-level simulation environment, in which the accuracy-energy trade-off can be easily performed to evaluate the energy consumption of the proposed MCU architecture.

Figure 10(d) shows energy consumption reduction according to the accuracy by configuring the S2E for specific signal segments. Using a timer and oscillator unit with $10 \%$ accuracy error in the swipe-gesture recognition application, the implemented MCU could still identify the gesture event, although consuming only $20 \%$ energy compared to the result of the accurate discrete time sampling method.

The elapsed time resolution for the time quantization reduces directly the power consumption, which is constantly required to monitor the incoming signal shape. Trade-off between the time quantization error and the power consumption reduction is performed to determine the error bound allowing the appropriate signal detection. The event segmentation size also affects the power consumption reduction slightly, which is showed with an example of 168 events and 104 events in Figure 10(d). The power consumption reduction 


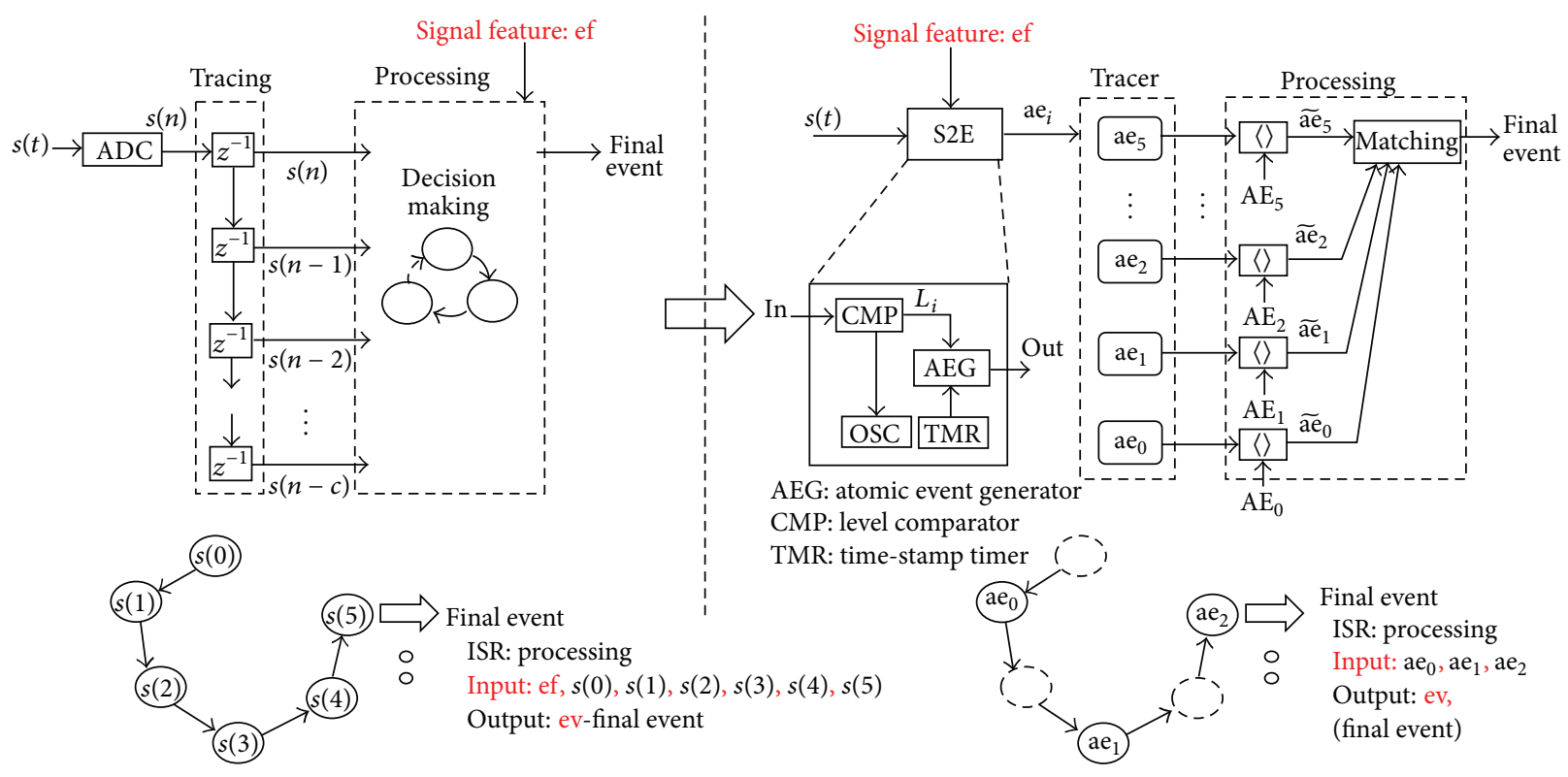

FIGURE 8: Comparison of conventional digital signal processing versus event-driven signal processing.

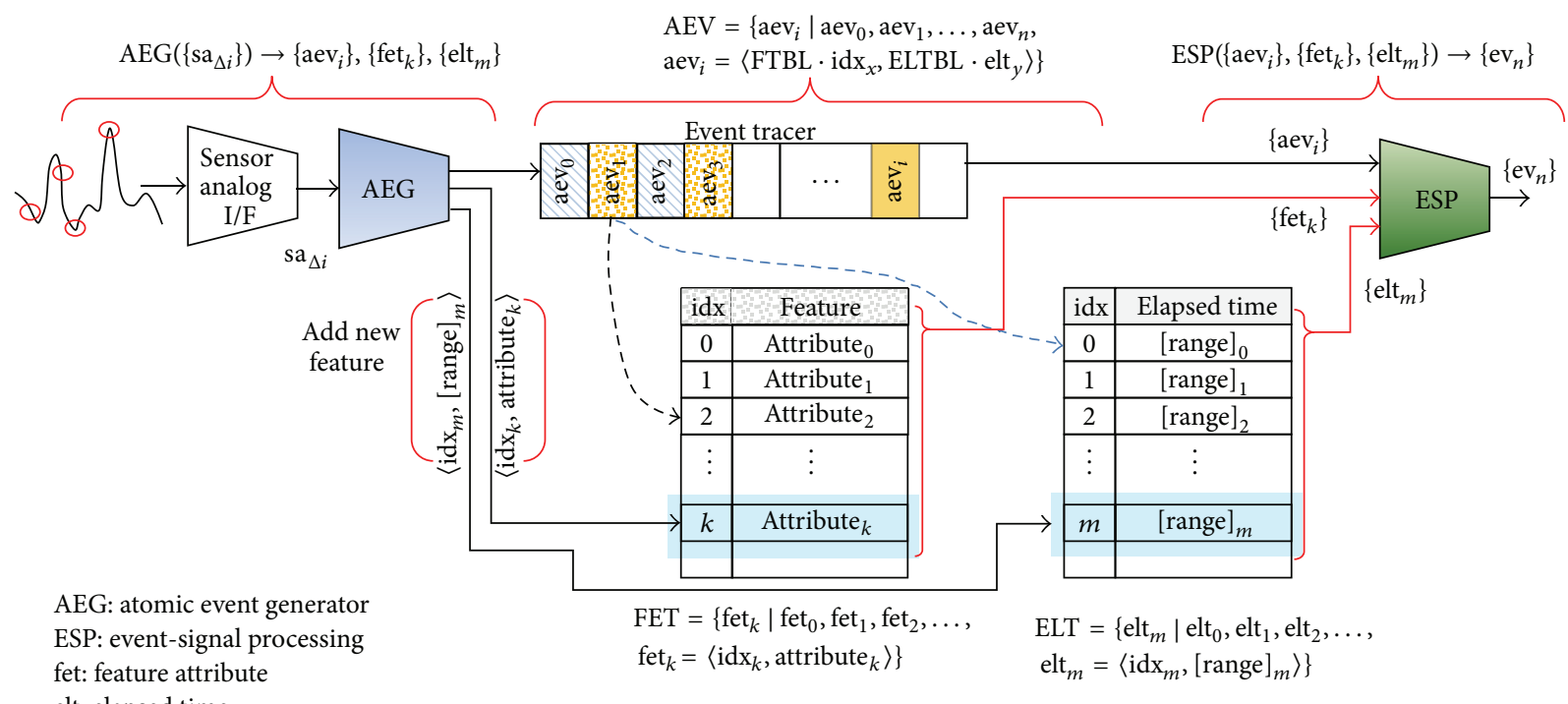
elt: elapsed time

FIGURE 9: Index-based feature table including attributes and elapsed time range.

is dependent on the event-quantization accuracy controlled by time measurement resolution and event segmentation size.

\section{Conclusion}

The macrolevel signal processing concept is based on the early evaluation of incoming sensor signal data by the S2E. The signal-specific signal segmentation with the features of interest enables the atomic event extraction from the continuous sensor data signal. The early evaluation of the signal features enables the entire system in sleep mode, with the exception of the S2E, to consume relatively little current. The extracted small number of atomic events is analyzed by the EPU, which will traverse the reduced state space. The proposed method requires the additional hardware by modifying the conventional MCU bus architecture and the user must perform the iterative configuration on the S2E and EPU carefully after analyzing the signal characteristics for rare-event activitysensing applications until the reasonable power reduction is accomplished. The event-space representation and signal abstraction of atomic events extracted by S2E could reduce the data processing cost in terms of the energy consumption 


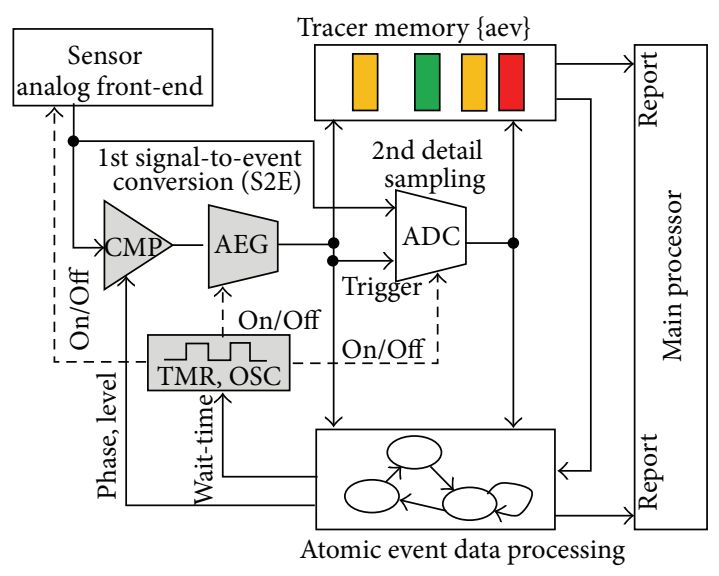

(a) S2E circuit data path

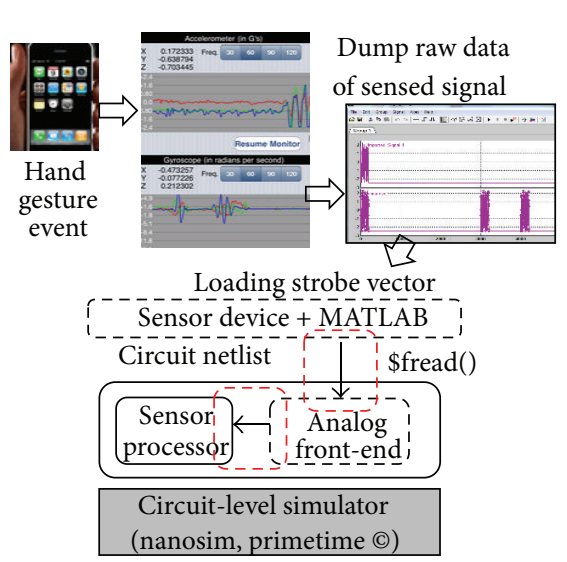

(c) Measurement environment

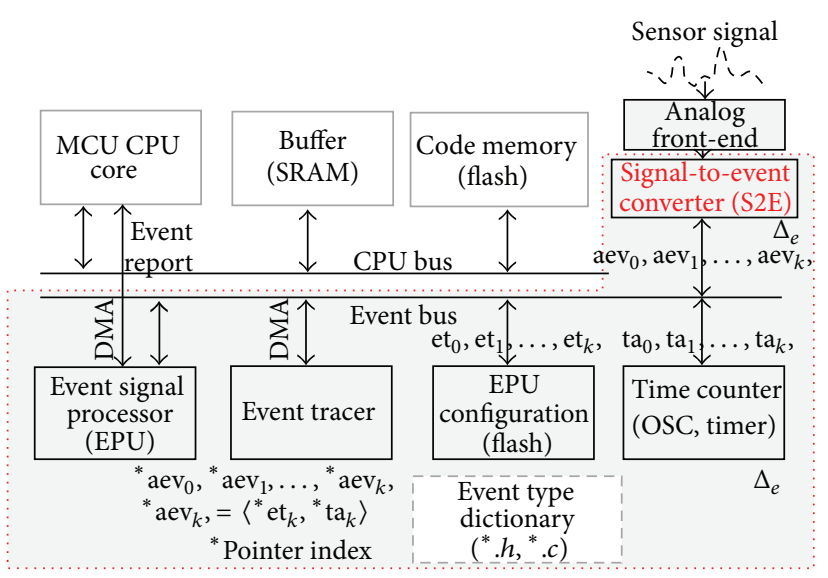

(b) Modified microcontroller bus architecture

Energy consumption and lifetime comparison: time-stamps measurement (OSC + time) current sweep

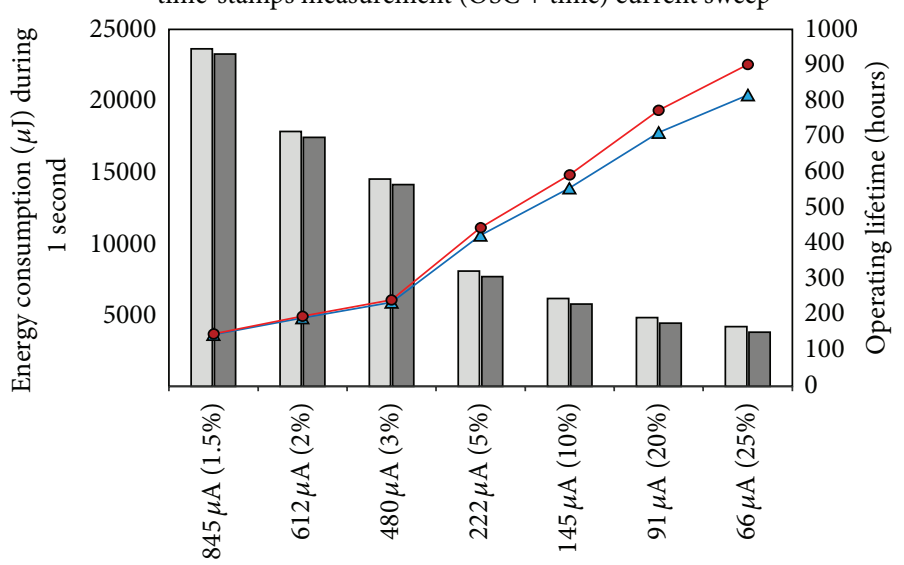

Operating current and accuracy of time quantizer block

$\sqsubset$ Energy (168 events/s) $\triangle$ Lifetime (168 events/s)

$\square$ Energy (104 events/s) $\rightarrow$ Lifetime (104 events/s)

(d) Energy consumption according to event quantization error

FIGURE 10: Implemented circuit and experimental results.

by considering specific characteristics of signals observed in rare-event sensing applications. The experimental result shows that the proposed method is an effective way to provide the power reduction.

\section{Conflict of Interests}

The authors declare that there is no conflict of interests regarding the publication of this paper.

\section{Acknowledgments}

This research was supported by Basic Science Research Program through the National Research Foundation of Korea (NRF) funded by the Ministry of Education (2014R1A6A3A04059410), the MSIP (Ministry of Science, ICT \& Future Planning), Korea, under the C-ITRC (Convergence Information Technology Research Center) support program (NIPA-2014-H0401-14-1004) supervised by the NIPA (National IT Industry Promotion Agency), and the 2013 Yeungnam University Research Grant.

\section{References}

[1] L. Chen, J. Hoey, C. D. Nugent, D. J. Cook, and Z. Yu, "Sensorbased activity recognition," IEEE Transactions on Systems, Man and Cybernetics Part C: Applications and Reviews, vol. 42, no. 6, pp. 790-808, 2012.

[2] Ó. D. Lara and M. A. Labrador, "A survey on human activity recognition using wearable sensors," IEEE Communications Surveys and Tutorials, vol. 15, no. 3, pp. 1192-1209, 2013.

[3] K. Van Laerhoven, H.-W. Gellersen, and Y. G. Malliaris, "Longterm activity monitoring with a wearable sensor node," in International Workshop on Wearable and Implantable Body Sensor Networks (BSN '06), pp. 171-174, April 2006.

[4] K. Leuenberger and R. Gassert, "Low-power sensor module for long-term activity monitoring," in Proceedings of the 33rd 
Annual International Conference of the IEEE Engineering in Medicine and Biology Society (EMBS '11), pp. 2237-2241, September 2011.

[5] Y. Tsividis, "Event-driven data acquisition and digital signal processing-a tutorial," IEEE Transactions on Circuits and Systems II: Express Briefs, vol. 57, no. 8, pp. 577-581, 2010.

[6] P. Panek, "Error analysis and bounds in time delay estimation," IEEE Transactions on Signal Processing, vol. 55, no. 7, pp. 35473549, 2007.

[7] Y. Tsividis, "Event-driven data acquisition and continuous-time digital signal processing," in Proceedings of the 32nd Annual Custom Integrated Circuits Conference (CICC '10), pp. 1-8, September 2010.

[8] B. Schell and Y. Tsividis, "Analysis of continuous-time digital signal processors," in Proceedings of the IEEE International Symposium on Circuits and Systems (ISCAS '07), pp. 2232-2235, May 2007.

[9] Y. Yilmaz, G. Moustakides, and X. Wang, "Spectrum sensing via event-triggered sampling," in Proceedings of the 45th Asilomar Conference on Signals, Systems and Computers (ASILOMAR '11), pp. 1420-1424, November 2011.

[10] M. Miśkowicz, “The event-triggered integral criterion for sensor sampling," in Proceedings of the IEEE International Symposium on Industrial Electronics (ISIE '05), vol. 3, pp. 10611066, June 2005.

[11] B. French, D. P. Siewiorek, A. Smailagic, and M. Deisher, "Selective sampling strategies to conserve power in context aware devices," in Proceedings of the 11th IEEE International Symposium on Wearable Computers (ISWC '07), pp. 77-80, October 2007.

[12] W. Li, M. Bandai, and T. Watanabe, "Tradeoffs among delay, energy and accuracy of partial data aggregation in wireless sensor networks," in Proceedings of the 24th IEEE International Conference on Advanced Information Networking and Applications (AINA '10), pp. 917-924, April 2010.

[13] K. Choi, R. Soma, and M. Pedram, "Fine-grained dynamic voltage and frequency scaling for precise energy and performance tradeoff based on the ratio of off-chip access to onchip computation times," IEEE Transactions on Computer-Aided Design of Integrated Circuits and Systems, vol. 24, no. 1, pp. 1828, 2005.

[14] Y. Lee, J. Kim, and C.-M. Kyung, "Energy-aware video encoding for image quality improvement in battery-operated surveillance camera," IEEE Transactions on Very Large Scale Integration (VLSI) Systems, vol. 20, no. 2, pp. 310-318, 2012.

[15] V. Gupta, D. Mohapatra, A. Raghunathan, and K. Roy, "Lowpower digital signal processing using approximate adders," IEEE Transactions on Computer-Aided Design of Integrated Circuits and Systems, vol. 32, no. 1, pp. 124-137, 2013.

[16] A. B. Kahng and S. Kang, "Accuracy-configurable adder for approximate arithmetic designs," in Proceedings of the 49th Annual Design Automation Conference (DAC '12), pp. 820-825, ACM, New York, NY, USA, June 2012. 

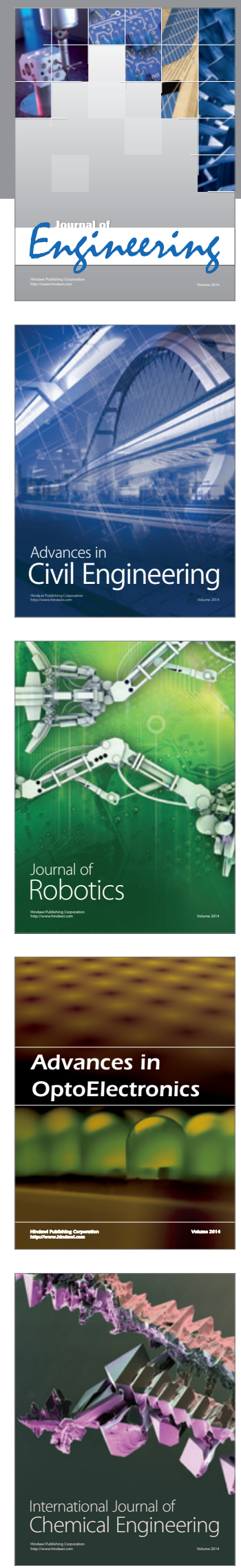

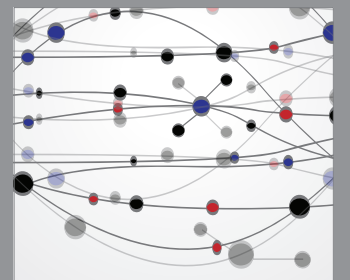

The Scientific World Journal
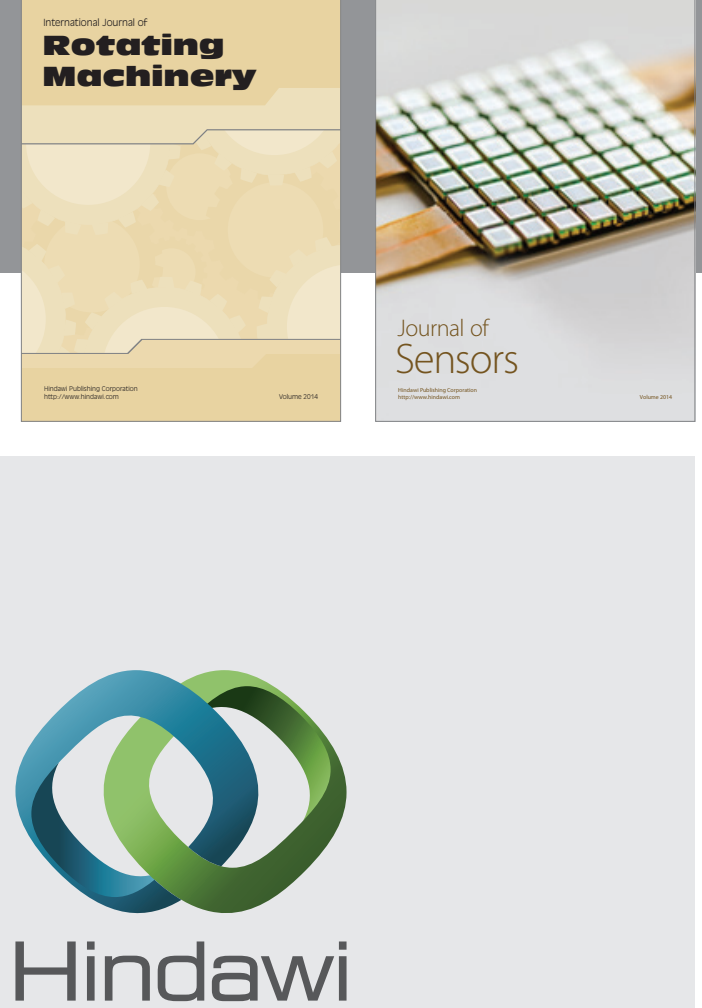

Submit your manuscripts at http://www.hindawi.com
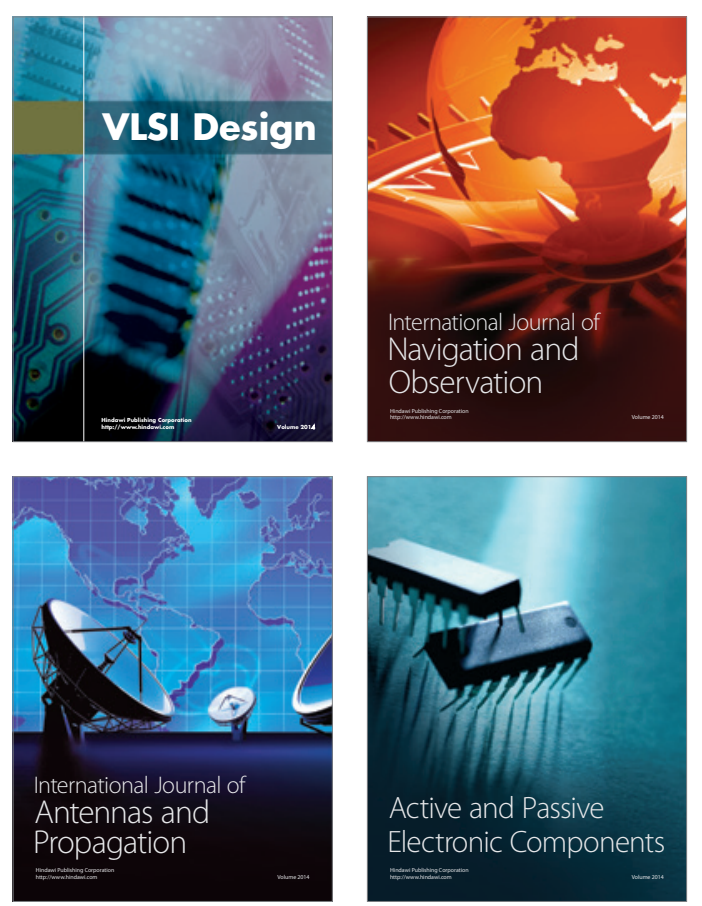
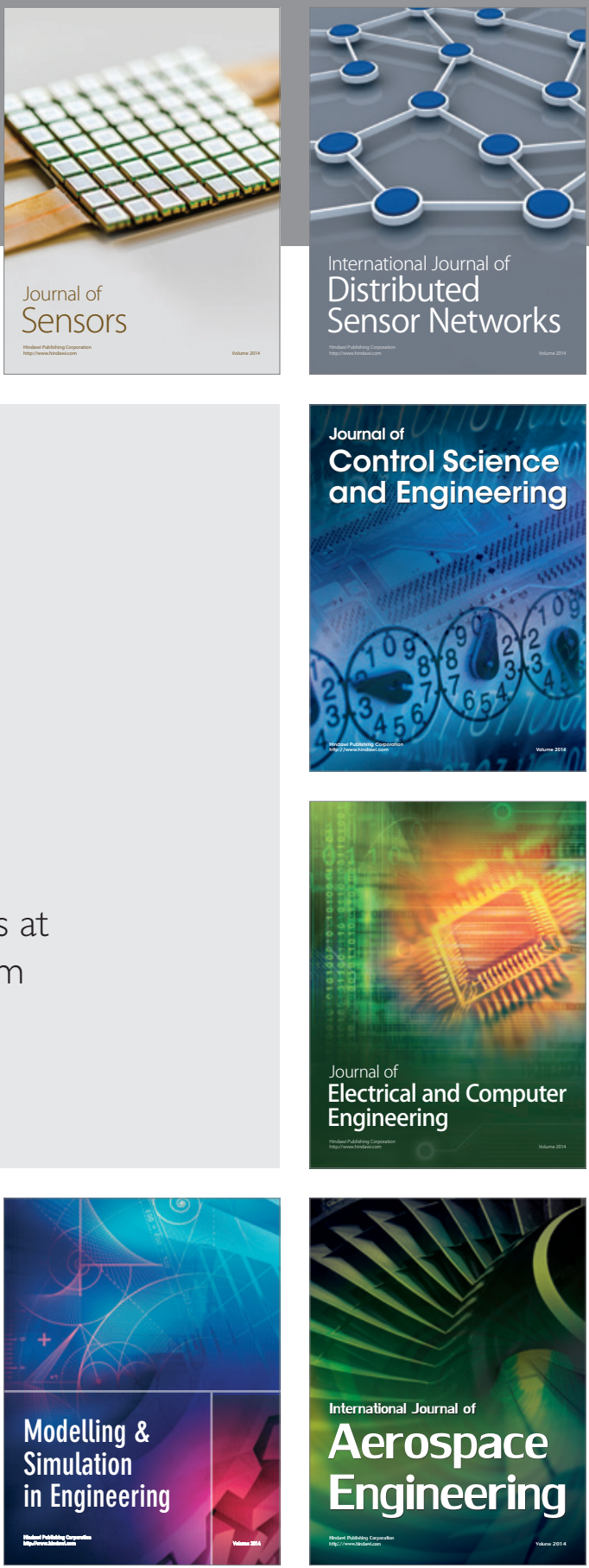

Journal of

Control Science

and Engineering
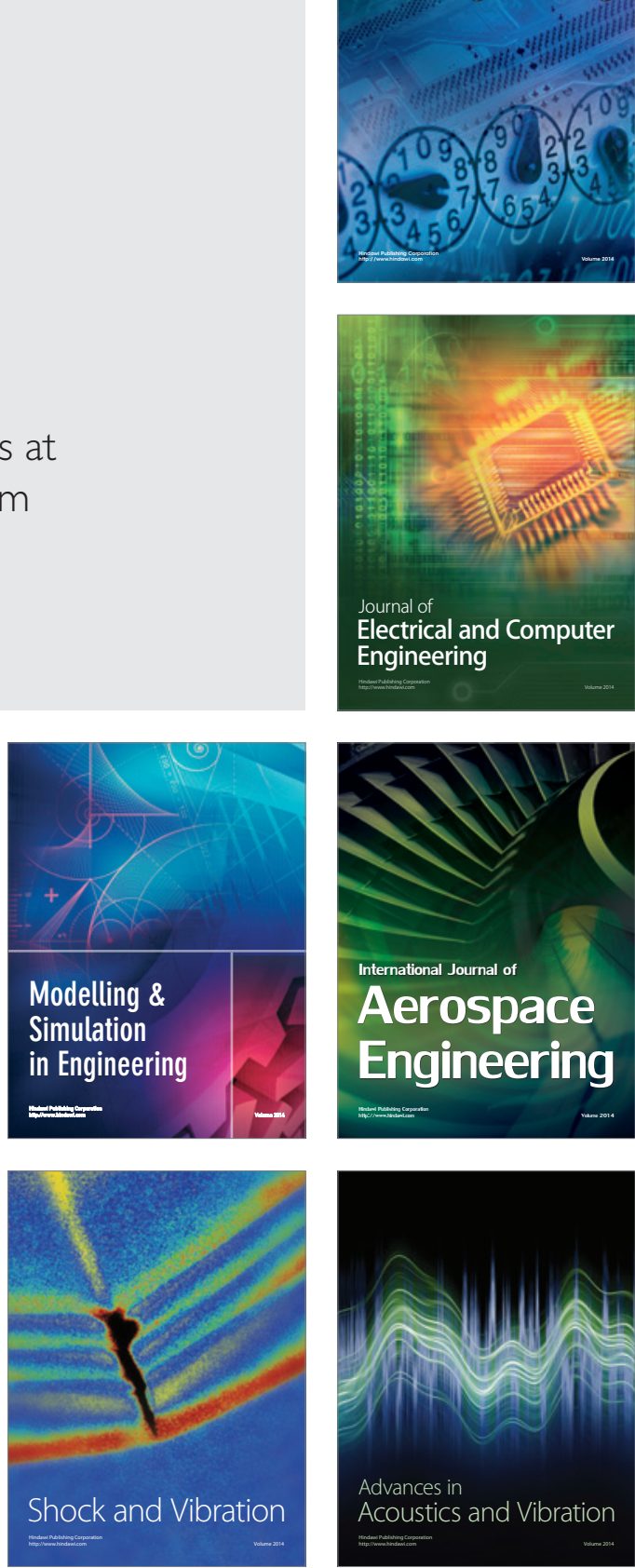